\title{
Diagnosis and management of hemoptysis
}

\author{
Anna Rita Larici, Paola Franchi, Mariaelena Occhipinti, Andrea Contegiacomo, Annemilia del Ciello, \\ Lucio Calandriello, Maria Luigia Storto, Riccardo Marano, Lorenzo Bonomo
}

\begin{abstract}
Hemoptysis is the expectoration of blood that originates from the lower respiratory tract. It is usually a self-limiting event but in fewer than $5 \%$ of cases it may be massive, representing a life-threatening condition that warrants urgent investigations and treatment. This article aims to provide a comprehensive literature review on hemoptysis, analyzing its causes and pathophysiologic mechanisms, and providing details about anatomy and imaging of systemic bronchial and nonbronchial arteries responsible for hemoptysis. Strengths and limits of chest radiography, bronchoscopy, multidetector computed tomography (MDCT), MDCT angiography and digital subtraction angiography to assess the cause and lead the treatment of hemoptysis were reported, with particular emphasis on MDCT angiography. Treatment options for recurrent or massive hemoptysis were summarized, highlighting the predominant role of bronchial artery embolization. Finally, a guide was proposed for managing massive and nonmassive hemoptysis, according to the most recent medical literature.
\end{abstract}

n clinical practice hemoptysis is a common symptom, which may require further investigation. It is defined as the expectoration of blood that originates from the lower respiratory tract (1). Bleeding from the upper airways is excluded from this definition.

In most cases hemoptysis is a self-limiting event but in fewer than $5 \%$ it may be severe or massive, representing a life-threatening condition that warrants urgent investigations and treatment (2). Massive hemoptysis usually refers to the expectoration of a large amount of blood and/ or to a rapid rate of bleeding. The blood volume expectorated over 24 hours is generally used for distinguishing massive and nonmassive hemoptysis, although the choice of a cutoff value is controversial (3). Volumes of 100 to $1000 \mathrm{~mL}$ of blood (4-9) have been described as indicative of massive hemoptysis, but no specific volume has been universally accepted. Furthermore, a large volume of expectorated blood alone should not define massive hemoptysis, but rather an amount of blood sufficient to cause a condition that threatens the patient's life can be a more correct and functional definition of severe hemoptysis $(4,5)$.

Asphyxia due to the flooding of the airways rather than exsanguination is usually the cause of death, and it is commonly accompanied by cardiovascular collapse. The mortality rate from untreated massive hemoptysis is more than $50 \%$ (6). Therefore, prompt recognition of severe hemoptysis and identification of its causes are mandatory to initiate an adequate treatment and to avoid fatal complications (6). Imaging plays a relevant role in managing this clinical condition.

This article aims to provide a comprehensive review on massive and nonmassive hemoptysis, with particular emphasis on the pathophysiologic mechanisms, the anatomy of systemic and pulmonary arteries responsible for hemoptysis, and the role of imaging modalities in diagnosing causes and helping treatment. Strengths and limitations of the various diagnostic modalities will be analyzed and a guide for managing hemoptysis, according to the most recent medical literature, will be proposed.

\section{Causes and pathophysiology}

Hemoptysis has multiple causes usually categorized under parenchymal diseases, airway diseases, and vascular diseases.

Bleeding may originate from small or large lung vessels (10). Bleeding from the small vessels usually causes a focal or diffuse alveolar hemorrhage and is mainly due to immunologic, vasculitic, cardiovascular, and coagulatory causes (Table 1). Causes of bleeding from the large vessels include infectious, cardiovascular, congenital, neoplastic, and vasculitic diseases (Table 2). However, the most frequent diseases causing hemop- larici@rm.unicatt.it, P.E, M.O., A.C., A.D.C., L.C., R.M. $\triangle$ ann

A. Gemelli Hospital, Catholic University, Rome, Italy; Bracco

Diagnostics Inc. (M.L.S.), Princeton, New Jersey, USA.

Received 23 October 2013; revision requested 18 November 2013; revision received 18 December 2013; accepted 24 December 2013.

Published online 30 April 2014.

DOI 10.5152/dir.2014.13426 
Table 1. Causes of hemoptysis from small vessels

\begin{tabular}{ll}
\hline Immunologic and vasculitic diseases & Acute lung allograft rejection \\
& Antiphospholipid antibody syndrome \\
& Behçet disease \\
& Goodpasture's syndrome \\
& Henoch-Schönlein purpura \\
& Isolated pulmonary capillaritis \\
& Microscopic polyarteritis \\
& Mixed cryoglobulinemia \\
& Wegener granulomatosis \\
& Mitral stenosis \\
Cardiovascular diseases & latrogenic (anticoagulants/thrombolytic agents) \\
Coagulatory diseases & Coagulopathies \\
& Diffuse alveolar damage \\
Others & Lymphangioleiomyomatosis \\
& Pulmonary capillary hemangiomatosis \\
& Pulmonary hemosiderosis \\
& Tuberous sclerosis \\
& Veno-occlusive disease
\end{tabular}

tysis are bronchiectasis, tuberculosis, fungal infections, and cancer $(4,7)$.

Two arterial vascular systems supply blood to the lungs: the pulmonary arteries and the bronchial arteries. The pulmonary arteries provide $99 \%$ of the arterial blood to the lungs and are involved in the gas exchange. The bronchial arteries supply nourishment to the extra- and intrapulmonary airways and to the pulmonary arteries (vasa vasorum), without being involved in the gas exchange (8). Mediastinal lymph nodes and nerves, visceral pleura, esophagus, vasa vasorum of the aorta, and pulmonary veins are also provided by the bronchial arteries (4).

Complex capillary anastomoses exist between the pulmonary arteries and the systemic bronchial arteries (9). When pulmonary circulation is compromised (e.g., in thromboembolic disease, vasculitic disorders, or in hypoxic vasoconstriction), the bronchial supply gradually increases causing a hyperflow in the anastomotic vessels, which become hypertrophic with thin walls and tend to break into the alveoli and bronchi, giving rise to hemoptysis. Likewise, in chronic inflammatory disorders, such as bronchiectasis, chronic bronchitis, tuberculosis, mycotic lung diseases, and lung abscess, as well as in neoplastic diseases, the release of angiogenic growth factors promote neo- vascularization and pulmonary vessel remodeling, with engagement of collateral systemic vessels (11). These new and collateral vessels are fragile and prone to rupture into the airways.

In cases of severe hemoptysis requiring treatment, the source of bleeding originates from bronchial and pulmonary arteries in $90 \%$ and $5 \%$ of cases, respectively (5). In the remaining 5\% of cases, hemoptysis may derive from nonbronchial systemic arteries (4). Very rarely, hemoptysis has been reported originating from pulmonary and bronchial veins $(12,13)$ and capillaries (14). A recent study by Noë et al. (15) shows that bleeding from bronchial arteries can coexist with bleeding from nonbronchial and pulmonary arteries in the same patient.

According to different authors, etiology of hemoptysis cannot be determined in $3 \%$ to $42 \%$ of cases and it is defined as cryptogenic $(7,16,17)$. Nevertheless, it has been demonstrated that a proportion of patients presenting with hemoptysis without any morbidity are smokers, and bleeding in smokers should be defined as smoke-related (occurring as a result of tobacco-induced bronchial wall inflammation), rather than cryptogenic (17). Moreover, with a more systematic use of chest computed tomography
(CT), particularly multidetector CT (MDCT), a decrease in the prevalence of hemoptysis without known cause might be expected (17).

\section{Anatomy of the lung arterial blood supply}

Bronchial arteries are the principal sources of hemoptysis amenable to treatment (5). Searching the bronchial arteries origin before treatment is helpful, because over $30 \%$ have an abnormal origin that may lead to endovascular treatment failure. The bronchial arteries commonly originate from the upper portion of the descending thoracic aorta. The origin is defined orthotopic if the arteries arise from the descending aorta at the level of the vertebral bodies of T5-T6 (or at the carina). When the bronchial arteries originate at other levels, including aortic branches, they are referred to as ectopic (18). Ectopic bronchial arteries commonly arise from the inferior aspect of the aortic arch, subclavian artery, brachiocephalic trunk, thyrocervical trunk, internal mammary artery, costocervical trunk, pericardiophrenic artery, inferior phrenic artery, abdominal aorta, and coronary arteries $(4,6,18)$.

Cauldwell et al. (19) reported the most frequent types of origin of orthotopic bronchial arteries in a population of 150 adult cadavers (Fig. 1). Usually, two or three branches of the bronchial arteries run parallel with the major bronchi and generate a peribronchial plexus by anastomosing with each other (20). Arterioles from this plexus perforate the muscular layer and create a parallel plexus in the bronchial submucosa. In normal conditions the diameter of bronchial arteries is less than $1.5 \mathrm{~mm}$ at the origin and less than 0.5 $\mathrm{mm}$ more distally (21). They are usually considered hypertrophic and a potential source of hemoptysis when larger than $2 \mathrm{~mm}$ at the origin (22).

Hemoptysis may also arise from nonbronchial systemic arteries, which enter the pulmonary parenchyma through transpleural adhesions due to chronic inflammatory processes (tuberculosis, mycosis) or through pulmonary ligaments (20) and anastomose with the pulmonary arterial circulation (8). Nonbronchial arteries 
Table 2. Causes of hemoptysis from large vessels

\begin{tabular}{|c|c|}
\hline \multirow[t]{7}{*}{ Infectious diseases } & Abscess \\
\hline & Bronchitis (acute or chronic) \\
\hline & Bronchiectasis \\
\hline & Fungal infection \\
\hline & Parasitic infection \\
\hline & Pneumonia \\
\hline & Tuberculosis/nontuberculous mycobacteria \\
\hline \multirow[t]{9}{*}{ Cardiovascular diseases } & Arteriovenous malformation \\
\hline & Bronchial artery aneurysm \\
\hline & Bronchovascular fistula \\
\hline & Congestive heart failure \\
\hline & Pulmonary embolism/infarction \\
\hline & Pulmonary hypertension \\
\hline & Right-sided endocarditis \\
\hline & Thoracic aortic aneurysm rupture/dissection \\
\hline & Septic pulmonary embolism \\
\hline \multirow[t]{3}{*}{ Congenital diseases } & Cystic fibrosis \\
\hline & Pseudosequestation \\
\hline & Pulmonary artery atresia or stenosis \\
\hline \multirow[t]{3}{*}{ Neoplastic diseases } & Bronchial adenoma \\
\hline & Lung metastasis \\
\hline & Primary lung cancer \\
\hline \multirow[t]{4}{*}{ Vasculitic diseases } & Behçet disease/Hughes-Stovin syndrome \\
\hline & Lupus pneumonitis \\
\hline & Takayasu arteritis \\
\hline & Wegener's granulomatosis \\
\hline \multirow[t]{10}{*}{ Others } & Chronic obstructive airway disease \\
\hline & Drug \\
\hline & Foreign body \\
\hline & latrogenic (Swan-Ganz catheter) \\
\hline & Interstitial fibrosis \\
\hline & Lung contusion \\
\hline & Pulmonary endometriosis \\
\hline & Trauma \\
\hline & Dieulafoy's disease of the bronchus \\
\hline & Cryptogenic hemoptysis \\
\hline
\end{tabular}

most commonly originate from inferior phrenic arteries, musculophrenic and pericardiodiaphragmatic arteries, posterior intercostal arteries, thyrocervical trunk, internal mammary arteries, and subclavian arteries (4).

Pulmonary arterial origin of hemoptysis is possible (23). Persistent hemoptysis despite appropriate embolization of the systemic arteries suggests a pulmonary arterial source of bleeding. Khalil et al. (24) investigated the potential causes of pulmonary arterial hemoptysis, including diseases causing necrosis (active tuberculosis, pulmonary abscess, aspergillosis, and necrot- imaging modality for evaluating a patient with hemoptysis $(1,6,8,10)$. It is quick, inexpensive, and readily available. CXR can assist in lateralizing bleeding and reveal a focal or diffuse lung involvement. CXR may detect underlying parenchymal and pleural abnormalities (25), such as masses, pneumonia, chronic lung disease, atelectasis, cavitary lesion, and alveolar opacities due to alveolar hemorrhage (10). A localized finding may guide further evaluation better than a diffuse or bilateral lung involvement.

Nevertheless, the sensitivity of CXR in this context is not high. Hirshberg et al. (28) reported only 50\% positive diagnostic yield for CXR. Revel et al. (29) demonstrated that CXR may identify the bleeding site in $46 \%$ of cases and the bleeding cause in 35\% only. In a study by Herth et al. (30), nearly a quarter of patients presenting with hemoptysis due to malignancy showed normal CXR. Therefore, in patients presenting with hemoptysis, a negative CXR warrants other diagnostic studies, including bronchoscopy and/ or MDCTA.

\section{Bronchoscopy}

For many years bronchoscopy has been considered the primary method for diagnosing and localizing hemoptysis, especially if massive (31).

Bronchoscopy, performed with either a rigid or flexible endoscope, is helpful for identifying active bleeding and for checking the airways in patients with massive hemoptysis.

Although rigid bronchoscopy may have a role in massive hemoptysis due to its ability to maintain airway patency (6), flexible fiberoptic bronchoscopy has the advantage of being carried out at the patient's bedside without anesthesia and in the intensive care unit, and is therefore more frequently used (10).

The capability and success of bronchoscopy in localizing the bleeding site may vary according to the rate and severity of the hemorrhage (Fig. 2). Hirshberg et al. (28) found that bronchoscopy was more effective in finding the bleeding site in patients with moderate to severe hemoptysis (64\% and 67\%) than in those with mild hemoptysis (49\%). Nevertheless, severe hemorrhage can 


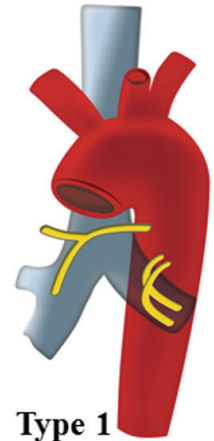

Type 1

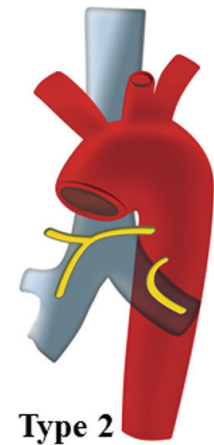

Type 2

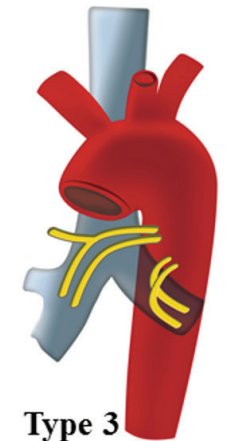

Type 3

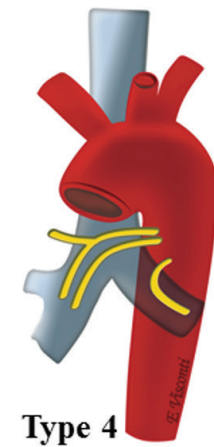

Type 4

Figure 1. Four most frequent origins and branching types of the orthotopic bronchial arteries at the level of tracheal bifurcation as described by Cauldwell et al. (19). The right bronchial artery usually originates from the intercostobronchial trunk (ICBT), which arises from the medial aspect of the descending aorta and also provides at least one right intercostal artery. The left bronchial artery commonly comes from the anterior aspect of the descending aorta. Type 1 is characterized by one right bronchial artery originating from the ICBT and two left bronchial arteries. Type 2 has one right bronchial artery from the ICBT and one left bronchial artery. Type 3 has two right bronchial arteries, including one from the ICBT and the other originating separately from the aorta, and two left bronchial arteries. Type 4 has two right bronchial arteries, as type 3, and one left bronchial artery. Scheme modified with the permission by Emiliano Visconti, MD, from Yoon W, Kim JK, Kim YH, et al. Bronchial and nonbronchial systemic artery embolization for life-threatening hemoptysis: A comprehensive review. RadiGraphics 2002;22:1395-1409.
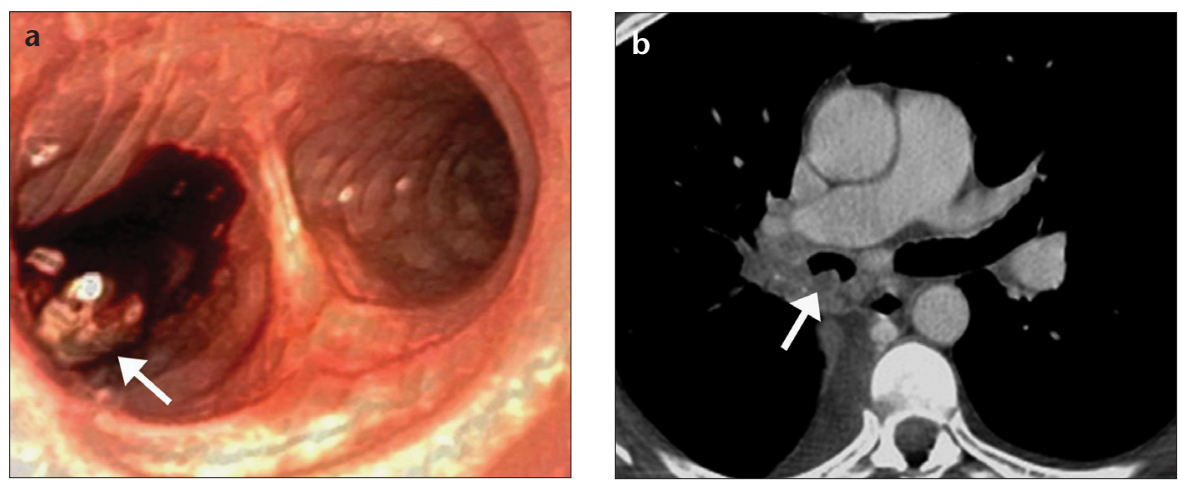

Figure 2. a, b. Flexible fiberoptic bronchoscopy (a) performed in a patient presenting with massive hemoptysis, showing the carina with active bleeding from an endoluminal lesion (arrow) located distally in the right main bronchus. Axial MDCT image at mediastinal window setting (b) demonstrates a soft tissue in the right hilum, abrupting into the lumen of distal main bronchus (arrow) and representing a squamous cell carcinoma. Right pleural effusion is also evident.

hinder the visualization of airways, in particular at the level of distal bronchi. Another limitation of bronchoscopy is that lavage or the use of endoscope itself may cause bronchial mucosa irritation and recurrent bleeding (8).

Bronchoscopy has an overall lower sensitivity than MDCT in detecting the underlying causes of bleeding $(8,25-27$, 29). In a study by Revel et al. (29), the cause of bleeding was identified in $8 \%$ of the patients, with bronchoscopy and in 77\%, with MDCT. Nevertheless, bronchoscopy yields additional information on endobronchial lesions and

allows samples for tissue diagnosis and microbial cultures (10). Moreover, with bronchoscopy cold saline solution can be instilled directly into the airways at the level of the bleeding source, if identified, and balloon inflation or laser coagulation may be used to control hemorrhage, even though the efficacy of these procedures is not proved and depends mostly on the practitioner's skill (32).

A recent study by Lee et al. (33) demonstrated that bronchoscopy has a relevant role mainly in patients with hemoptysis and no explainable lesions on MDCT.

\section{Multidetector CT}

MDCT represents a noninvasive and highly useful imaging tool in the clinical context of hemoptysis and allows a comprehensive evaluation of the lung parenchyma, airways, and thoracic vessels by using contrast material.

MDCT may identify the bleeding site in $63 \%$ to $100 \%$ of patients with hemoptysis $(25,34)$ and has the ability to uncover the potential underlying causes of bleeding, such as bronchiectasis, pulmonary infections, lung cancer, etc., being superior to bronchoscopy in this respect. MDCT also has the advantage of showing distal airways beyond the level of the bronchoscope $(25,26$, 28,33 ), and it has a sensitivity of more than $90 \%$ in identifying endobronchial lesions (10).

Nevertheless, there are some limitations in characterizing lesions such as endobronchial blood clots that may mimic a tumor and visualizing an endobronchial process in the presence of acute bleeding filling the bronchial lumen (35). In these cases bronchoscopy remains an important complementary diagnostic tool (10). It has been affirmed that the combined use of MDCT and bronchoscopy provides the best accuracy in assessing patients with hemoptysis (34). Numerous authors have suggested that MDCT should be carried out before eventual bronchoscopy in all patients with hemoptysis $(5,27)$.

MDCTA is useful to create a detailed and accurate map of the thoracic vasculature that may guide further treatment. Remy-Jardin et al. (36) demonstrated that MDCTA provides more detailed depiction of bronchial and nonbronchial systemic arteries than DSA.

In cases of hemoptysis, vessel analysis should include the bronchial and nonbronchial arteries and the pulmonary arterial circulation. A MDCTA of the chest performed before treatment is of value because the number and the origin of bronchial arteries may be carefully evaluated and the coexistence of an additional nonbronchial arterial supply may be easily depicted to determine the optimal angiographic approach $(4,36)$. Recognition of more than one arterial supply before endovascular treatment in patients presenting with massive hemoptysis, will assist in choosing ectopic vessels 
amenable to embolization and in preventing recurrences of hemoptysis after initial successful embolization (15). The availability of this information before patient's arrival in the angiographic suite reduces the procedure time and potential iatrogenic risks of searching for abnormal vessels, which is particularly important for unstable patients, and minimizes the contrast load and fluoroscopy radiation dose for patients and operators (15).

In summary, for a comprehensive assessment of the potential causes of hemoptysis it is advisable to perform a MDCTA (8).

\section{Multidetector CT angiography technique and imaging findings}

MDCT allows the scan of a wide anatomic volume over a short period of time with high spatial resolution, leading to reduction of the respiratory motion artifacts and increase in the image quality (8).

A MDCTA of the thorax can be carried out during a single breath hold in most patients by using a 16 and above detector row scanners and contrast material. A contrast-enhanced study is mandatory for identifying pulmonary and systemic arterial vessels. Image acquisition should extend from the inferior portion of the neck to include the supra-aortic great vessels, to the level of renal arteries, corresponding approximately to the vertebral body of L2, to include the infradiaphragmatic arteries.

Optimal enhancement of arteries may be achieved with intravenous injection of high-density contrast material $(350-400 \mathrm{mgl} / \mathrm{mL})$ at the flow rate of 3.5 to $5 \mathrm{~mL} / \mathrm{s}$, by using an automated injector through an 18-gauge cannula into an antecubital vein $(8,36)$. A region of interest should be positioned on the descending aorta. The scan should start during the peak enhancement (greater than $100 \mathrm{HU}$ ) of arterial phase. Thin collimation $(16 \times 0.75 \mathrm{~mm}$; $64 \times 0.6 \mathrm{~mm} ; 128 \times 0.6 \mathrm{~mm} ; 2 \times 128 \times 0.6$ $\mathrm{mm})$ and pitch adjusted to cover the entire chest during a single breath-hold should be used to acquire images (8).

MDCTA produces high-resolution angiographic studies complemented by high quality reformatted images at mediastinal window setting that allow the identification of origin and course
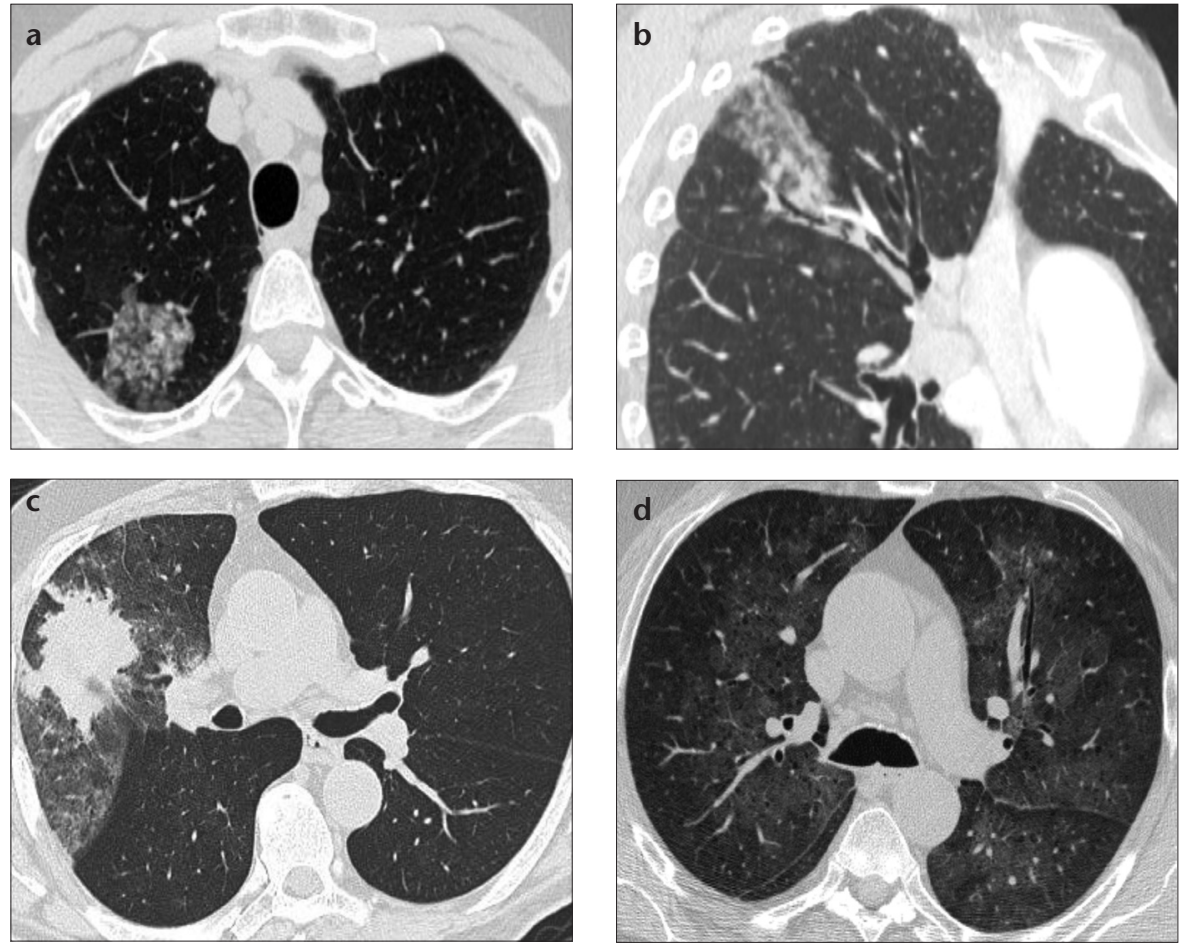

Figure 3. a-d. Focal and diffuse pulmonary hemorrhage. Axial thin-section MDCT image at parenchymal window setting (a) shows a focal well-defined intra-alveolar ground-glass area located in the posterior segment of the right upper lobe in a smoker patient with hemoptysis, representing parenchymal hemorrhage. Oblique paracoronal reconstruction (b) depicts the continuity between the lung abnormality and the posterior segmental and subsegmental bronchi filled with intraluminal material, likely indicative of blood. Axial thin-section MDCT image at parenchymal window setting (c) demonstrates a right upper lobe non-small cell lung cancer surrounded by localized hazy ground-glass opacity, indicating pulmonary hemorrhage secondary to tumor bleeding. Diffuse pulmonary hemorrhage (d) in a patient with atrial fibrillo-flutter treated with oral anticoagulant drugs, appearing as bilateral parahilar ground-glass attenuation without distortion of the lung parenchyma, associated with mild centrilobular emphysema.

of the arteries causing the bleeding. Axial thin-section images are useful in depicting the origin of bronchial arteries. Two-dimensional multiplanar and maximum intensity projection (MIP) reformations, including oblique planes, are accurate in providing images of the sinuous course of bronchial and nonbronchial arteries from their origins to the lungs better than axial images. The section thickness and the obliquity degree of the reformatted images should be adapted to the individual case to accurately represent the vessels involved. Three-dimensional volume rendering "road maps" of the hypertrophic vessels are very useful to interventional radiologists and surgeons in planning the correct treatment.

When performing a MDCTA of the chest in patients with hemoptysis, radiologists should look for the possible cause of bleeding and localize the site of hemorrhage within the lung paren- chyma at thin-section images. Pulmonary hemorrhage usually appears as focal or diffuse hazy consolidation or ground-glass opacity (Fig. 3) (8), even though thickened interlobular septa superimposed on a background of ground-glass attenuation ("crazy paving" pattern) have also been described. The evidence of material filling the lumen of bronchi may guide the identification of bleeding site (8).

A comprehensive review of contrast-enhanced images should include the assessment of normal or hypertrophic bronchial arteries into the mediastinum, by identifying their orthotopic or ectopic origin, their caliber, and course towards major bronchi. Hypertrophic nonbronchial systemic collaterals, as well as pulmonary arteries should also be carefully investigated to assess their potential contribution to bleeding.

Bronchial arteries with a normal diameter may be visualized at MDCTA 
(Fig. 4), as reported in a study by Morita et al. (37), where 97\% (217/223) of normal bronchial arteries were successfully identified in patients undergoing thoracoscopic surgery for esophageal cancer treatment.

Orthotopic bronchial arteries are identified on axial images, particularly when hypertrophic, as clusters of enhancing nodules or lines in the mediastinum, at the level of or below the aortic arch, usually connected with the descending aorta (Fig. 5a, 5b). Two dimensional MIP and three-dimensional volume rendering images (Fig. 5c, 5d) accurately delineate the anatomy of the bronchial arteries with their mediastinal and hilar course and allow the recognition of ectopic bronchial arteries (Fig. 6) (21, 36).

Although a diameter greater than 2 $\mathrm{mm}$ is usually considered abnormal and may be an indication for embolization (8), a poor correlation between bronchial arterial dilatation and risk of hemorrhage has been reported (38). The visibility of bronchial arteries from their origins to the hilum seems to be a more important finding than the vessel diameter. Yoon et al. (21) demonstrated that most bronchial arteries causing hemoptysis (74\%) were traceable at MDCTA, in contrast to those not causing hemoptysis (11\%).

Nonbronchial systemic arteries may be considered as causing hemoptysis when enlarged vascular structures within the extrapleural fat are shown in association with pleural thickening ( $\geq 3 \mathrm{~mm}$ ) and lung parenchyma abnormalities (39). Their route is not parallel to the bronchi and this feature distinguishes these vessels from ectopic bronchial arteries that always follow bronchial branches (Fig. 7) (20).

The pulmonary arteries should always be examined to exclude pulmonary embolism causing hemoptysis. Pulmonary arteries may also be responsible for bleeding in cases of arterial infiltration by neoplastic tissue (Fig. 8) and by necrotizing inflammatory disorders, such as tuberculosis (40). Pulmonary artery pseudoaneurysms can occur in association with lung abscesses, septic emboli, bronchiectasis, and lung neoplasms, as well as after trauma from Swan-Ganz catheter placement and in chronic cavitary tuberculosis
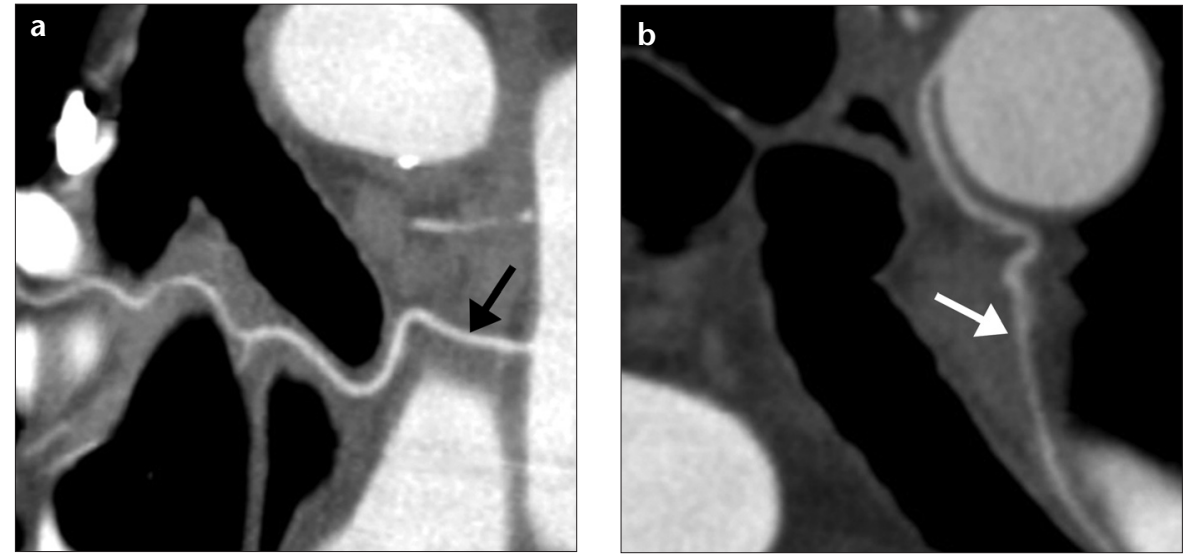

Figure 4. a, b. Normal orthotopic right and left bronchial arteries. Oblique MIP images at mediastinal window setting show one right (a) and one left (b) bronchial artery arising from the medial aspect of the thoracic descending aorta at the level of tracheal bifurcation. The right bronchial artery (black arrow) courses below the carina towards the right main bronchus, while the left bronchial artery (white arrow) runs parallel to the left main bronchus.
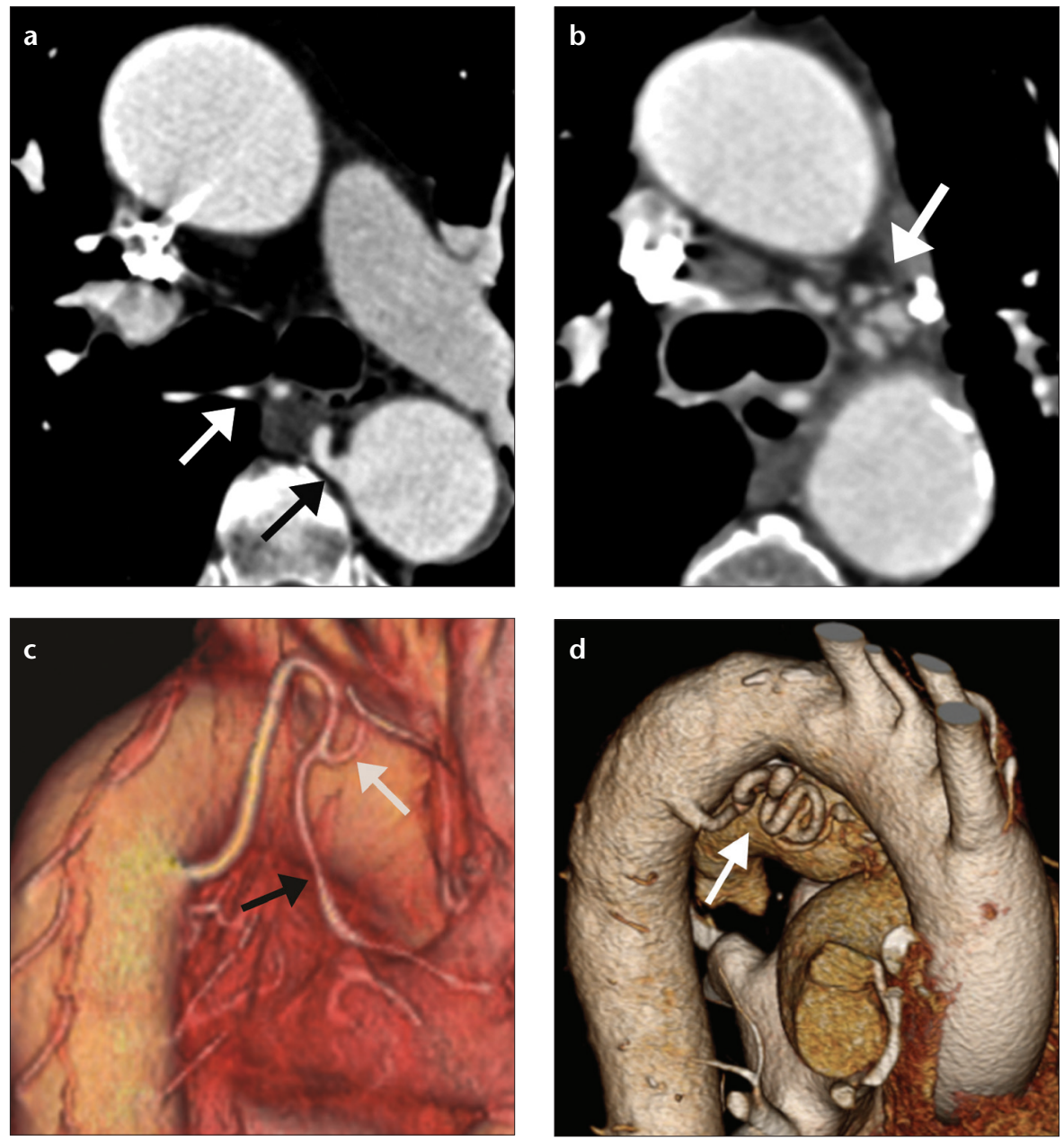

Figure 5. a-d. Hypertrophic orthotopic right and left bronchial arteries. Axial enhanced MDCT images at mediastinal window show right (a) and left (b) bronchial arteries as cluster of enhancing nodules below the aortic arch, behind the posterior wall of the main bronchi and in the aorto-pulmonary window (white arrows), connected with the descending aorta (a, black arrow). Three-dimensional volume rendering images $(\mathbf{c}, \mathrm{d})$ better depict on the right side (c) the presence of an intercostobronchial trunk that originates from the medial aspect of the descending aorta and has a cranial course before its bifurcation into one intercostal artery (white arrow) and one right bronchial artery (black arrow) with caudal course, and on the left side

(d) the short and tortuous course of the left bronchial artery in the aorto-pulmonary window towards the left hilum (white arrow). 

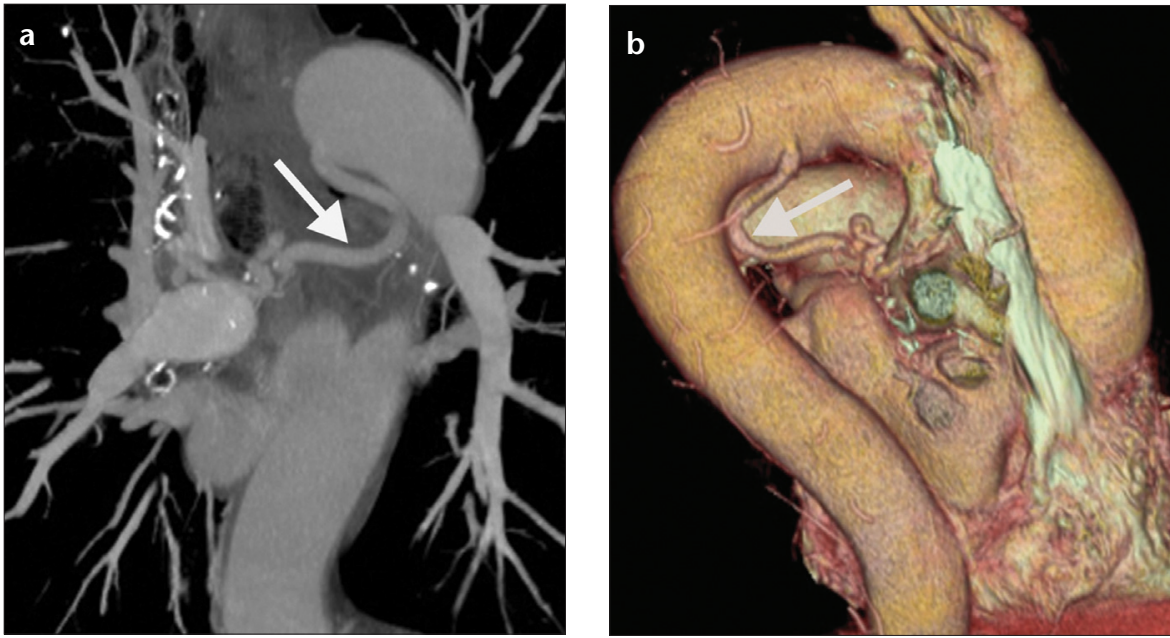

Figure 6. a, b. Hypertrophic ectopic right bronchial artery. Oblique MIP image at mediastinal window (a) and 3D volume rendering image (b) show the ectopic origin of a hypertrophic right bronchial artery arising from the concavity of the aortic arch and running towards the right hilum (arrows).
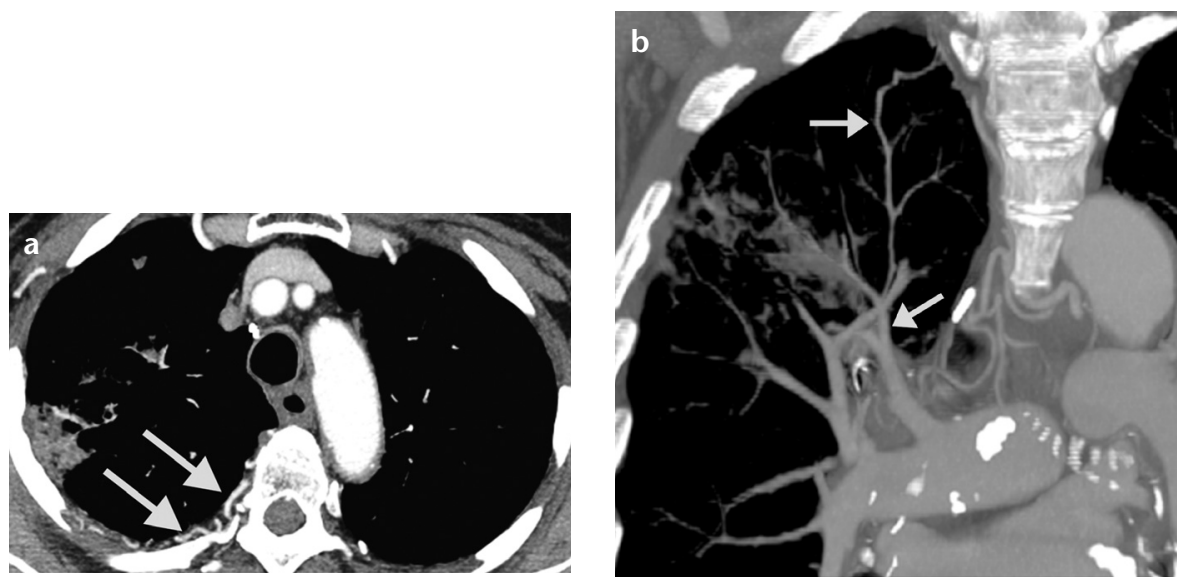

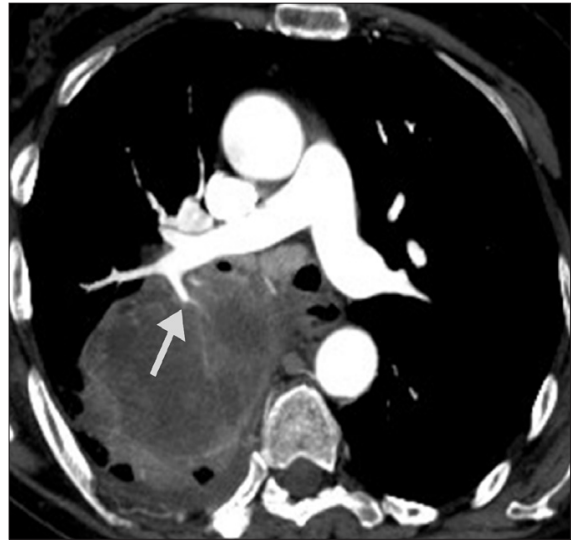

Figure 8. Pulmonary arterial bleeding. Contrast-enhanced axial CT image shows a large squamous cell carcinoma with extensive necrotic component, infiltrating the arterial branch of the apical segment of the right lower lobe (arrow) and the intermediate bronchus in a 55-year-old woman.

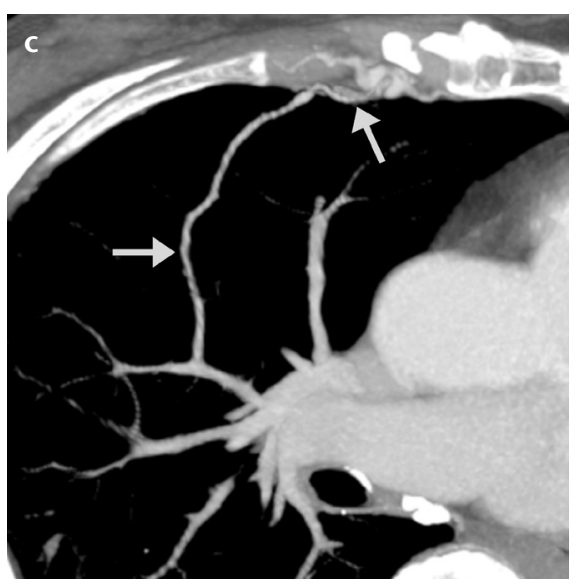

Figure 7. a-c. Hypertrophic nonbronchial arteries. Axial CT image at mediastinal window (a) of a 69-year-old woman with history of tuberculosis shows right upper lobe consolidation and enlarged and tortuous right upper intercostal artery (arrows) that courses along thickened pleura and goes towards the lesion. Coronal MIP image (b) detects a collateral hypertrophic nonbronchial vessel arising from the right intercostal artery that enters into the lung parenchyma with a vertical course (not parallel to the bronchi) and anastomoses with a pulmonary arterial branch (arrows). In the same patient, axial MIP reconstruction (c) demonstrates a hypertrophic nonbronchial arterial vessel originating from an enlarged right internal mammarian artery that enters into the lung parenchyma with a horizontal course and anastomoses with the arterial pulmonary system (arrows).

(Rasmussen's aneurysm) (40). The suspicion of a pseudoaneurysm should rise when an avidly enhancing nodule is identified within a lesion on contrast-enhanced CT scans.

Hemoptysis of pulmonary arterial origin may be associated with pulmonary artery aneurysm (Behçet disease) (41) or with PAVMs. Although pulmonary angiography has been considered the "gold standard" for diagnosis of PAVM, MDCTA has assumed a greater role in this regard (42). Interestingly, Remy et al. (43) found that contrast-enhanced CT has a better performance than pulmonary angiography in identifying PAVMs, with a sensitivity of $98.2 \%$ (vs. $59.6 \%$ of pulmonary angiography), and provides a reliable analysis of PAVM angioarchitecture that is very helpful in planning the treatment option, in particular for managing complex lesions.

\section{Digital subtraction angiography}

Nowadays DSA has a marginal role as a diagnostic tool in detecting the origin of hemoptysis.

Bronchial arteries are the main source of hemoptysis. The recognition of all the possible origins of the bronchial arterial supply at angiography may be challenging (15). Moreover, if angiography is performed as the first diagnos- tic step, nonbronchial or extrathoracic feeders may be missed or not sought as the main source of bleeding (15). Therefore, DSA is now reserved for cases where endovascular treatment has to be attempted and once the other diagnostic studies, such as MDCTA, have already been completed.

Bronchial artery embolization (BAE) is not free from complications. The most disastrous event is spinal cord ischemia. Its prevalence after BAE is between $1.4 \%$ and $6.5 \%$ according to different studies (44-47). Spinal cord ischemia can occur after inadvertent occlusion of the artery of Adamkiewicz, which supports the anterior 

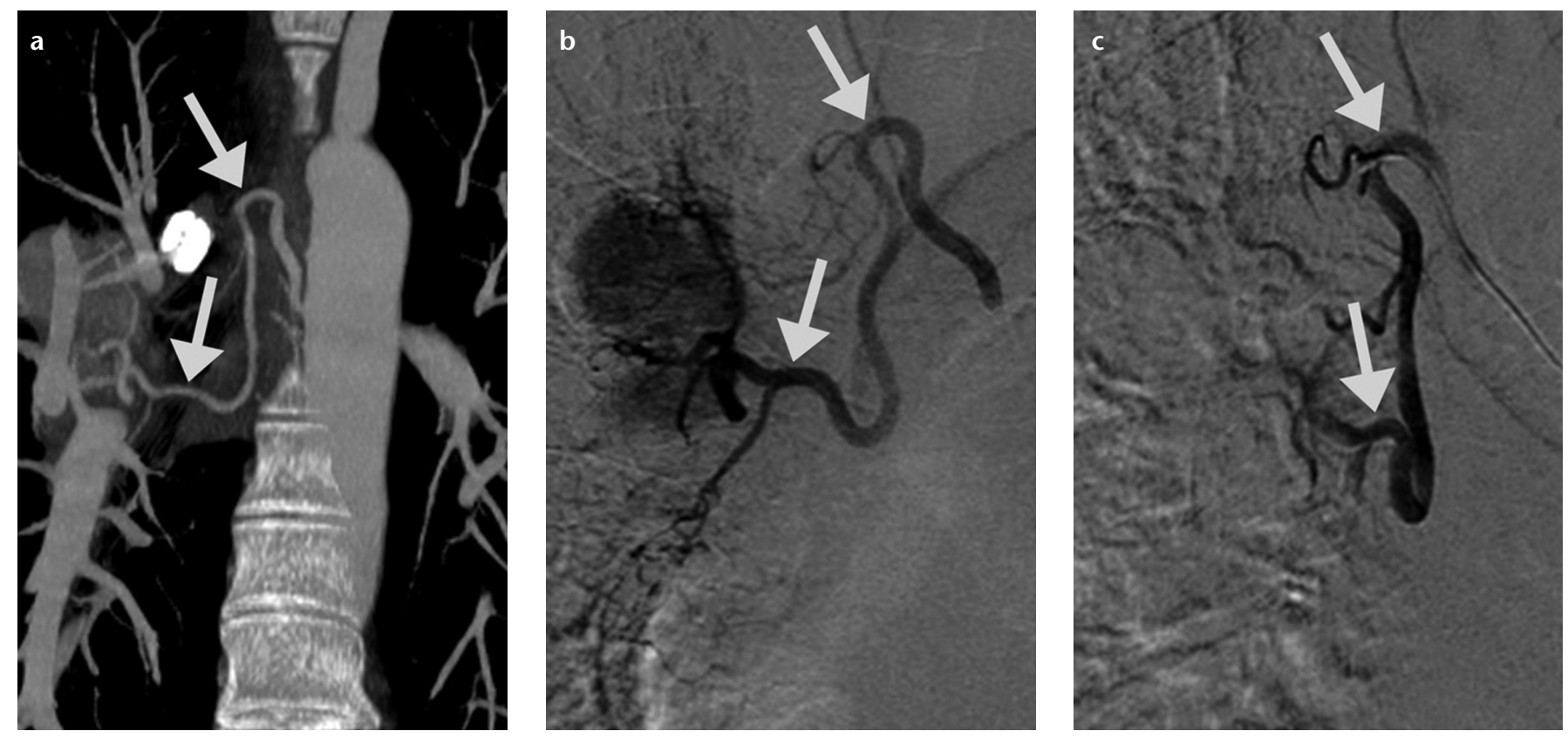

Figure 9. a-c. Oblique MIP image at mediastinal window setting (a) demonstrates a hypertrophic right bronchial artery (arrows) that supplies a pulmonary neuroendocrine mass in a 45-year-old man presenting with relapsing hemoptysis. Pre-embolization DSA (b) confirms the blood supply of the mass from the right bronchial artery (arrows). Postembolization image (c) shows the disappearance of lesion vascular supply (arrows). A subsequent resolution of hemoptysis was observed and eventually the patient underwent surgery.

spinal artery supply in the distal thoracic and lumbar regions. The Adamkiewicz artery originates from the descending thoracic aorta at the levels of T9-T12 in $75 \%$ of cases. However, it may originate between levels of $\mathrm{T} 5$ and T8 (48), and when this vessel is visualized at angiography, embolization should not be carried out (4). At MDCTA performed before the embolization, Adamkiewicz artery may be depicted (48), even though its fine caliber makes certain identification difficult. On the other hand, DSA is suitable in identifying this small vessel.

\section{Treatment}

Until twenty years ago, surgery was considered the treatment of choice for hemoptysis once the bleeding site was localized. However, surgery during an acute episode of hemorrhage implies a high risk of complications, with associated mortality rate ranging from 7\% to $18 \%$, which increases up to $40 \%$ in emergency (49). Moreover, not all patients are candidates for surgery, such as those with pre-existing respiratory and cardiovascular comorbidities.

Nowadays, surgery remains the treatment of choice only in selected cases, such as chest trauma and iatrogenic pulmonary artery rupture (5), while endovascular embolization is consid- ered the most effective and minimally invasive procedure for managing massive and recurrent hemoptysis in almost all other cases. Endovascular embolization may constitute a definitive therapy or it may be used as a tool to stabilize the patient before surgery $(5,7,50)$. Embolization reduces the pressure in the fragile hypertrophic arterial vessels supplying the pathological lung areas and decreases the risk of perioperative bleeding (Fig. 9) (6).

As the bronchial arteries are responsible for massive hemoptysis requiring treatment in over $90 \%$ of cases (8), $\mathrm{BAE}$ is the most frequently performed endovascular procedure. It promptly brings the bleeding under control in $66 \%$ to $90 \%$ of patients $(7,49)$.

Samara et al. (51) demonstrated that BAE is also an effective tool for managing massive cryptogenic hemoptysis. Menchini et al. (17) observed immediate cessation of bleeding in $85 \%$ of smoker patients with no other morbidities, undergoing DSA and BAE. Moderate to marked hypertrophic bronchial arteries were found in only $80 \%$ of these patients, while the remaining $20 \%$ had angiographically normal bronchial arteries on the bleeding side. This result confirms that normal bronchial arteries may also be responsible for bleeding in smokers.
Aside from the immediate control of bleeding, Anuradha et al. (52) recently demonstrated that in patients with massive hemoptysis due to tuberculosis and post-tuberculous sequelae, the effectiveness of BAE tends to decrease over years, being $51 \%$ in the first year and $39 \%$ in the second year after treatment. Nevertheless, repeated BAE in patients with early recurrence improves the outcome.

Also nonbronchial systemic arteries and pulmonary arteries may be subjected to an endovascular treatment, if they are the source of the hemorrhage $(15,53)$.

\section{Management}

In this review we attempted to create a possible algorithm for managing hemoptysis according to the available data in the medical literature.

Patients with hemoptysis should be managed based on the rate and severity of bleeding (massive or nonmassive) and the clinical condition of the patient. In case of massive bleeding in unstable patients, resuscitation is mandatory before any other diagnostic investigation.

\section{Massive hemoptysis}

The flowchart for massive hemoptysis is summarized in Fig. 10. An initial CXR is advisable in order to localize the bleeding site. MDCTA should be 


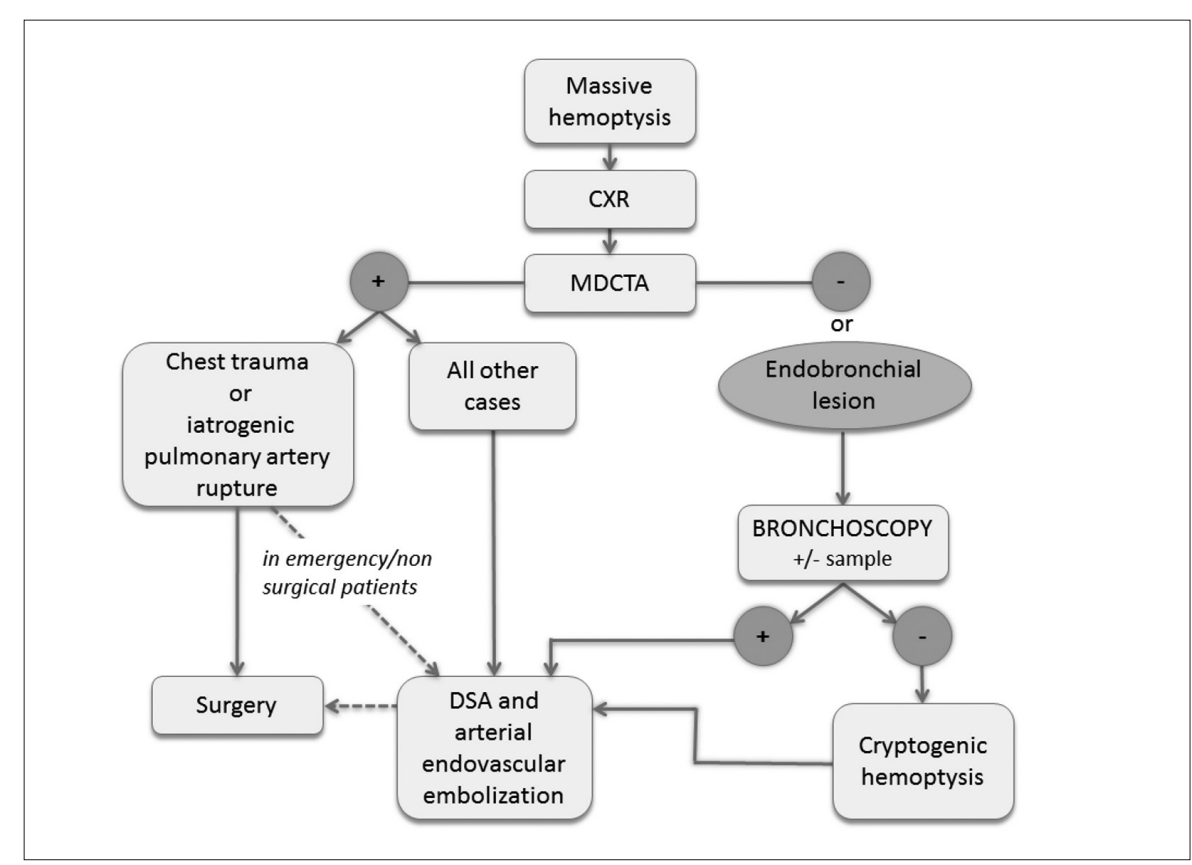

Figure 10. Flowchart for managing massive hemoptysis. CXR, chest radiography; MDCTA, multidetector computed tomography angiography; DSA, digital subtraction angiography.

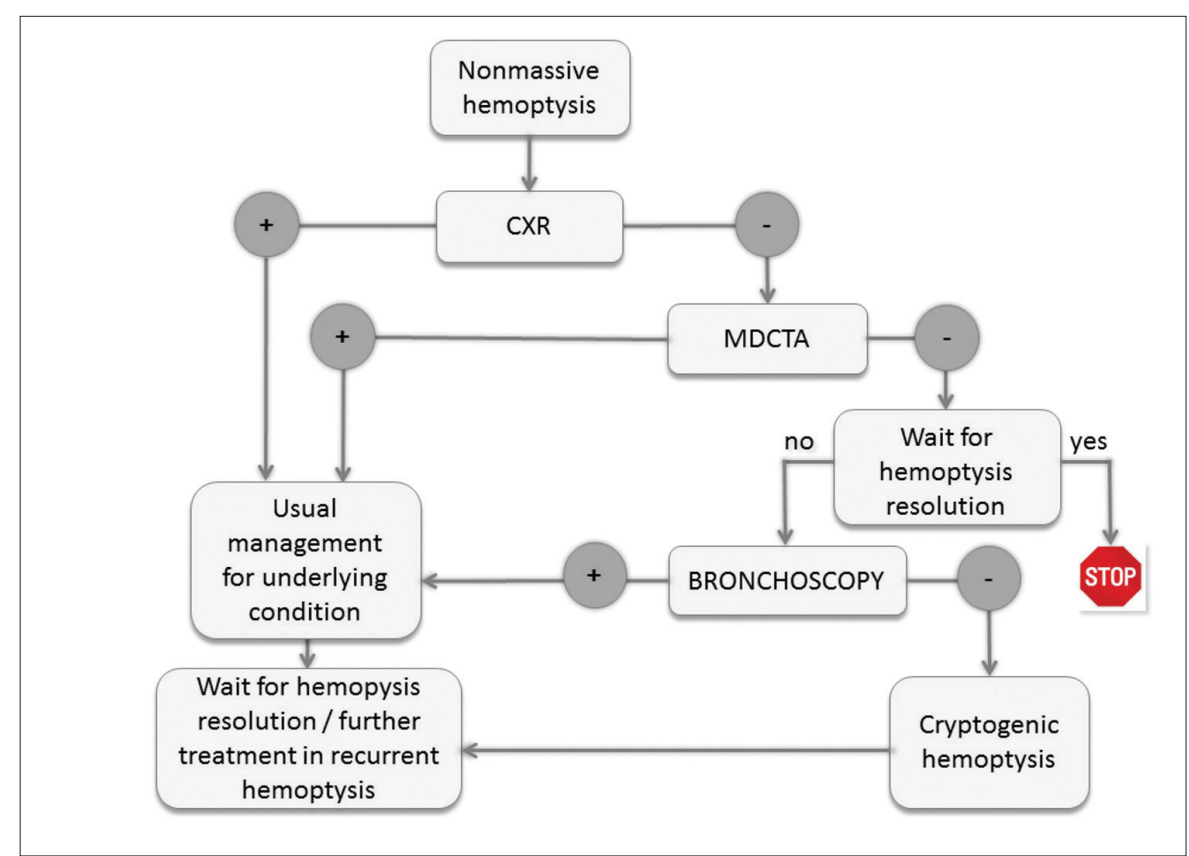

Figure 11. Flowchart for managing nonmassive hemoptysis. CXR, chest radiography; MDCTA, multidetector computed tomography angiography.

executed in any case, even in emergency, regardless of CXR results, due to its undisputed superiority in identifying the bleeding source and the possible underlying cause, allowing for better planning of further management and treatment $(30,32,35)$.

If the underlying cause is chest trauma or iatrogenic pulmonary artery rup- $\mathrm{cy}$, all patients with massive hemoptysis should undergo endovascular embolization regardless of the cause; definitive treatment through surgery may be performed eventually, once the patient is stabilized (49).

If MDCTA is negative or reveals an endobronchial lesion, bronchoscopy with eventual tissue sampling is recommended $(10,35)$. If bronchoscopy reveals the lesion, artery embolization should be the next step followed by surgery, if required. If bronchoscopy does not reveal any abnormality, a cryptogenic massive hemoptysis is diagnosed and embolization may be indicated, particularly in smokers (17).

\section{Nonmassive hemoptysis}

The flowchart for nonmassive hemoptysis is summarized in Fig. 11. A CXR should always be performed as a first instance exam $(1,6,8,10)$. If the underlying parenchymal or pleural abnormalities causing hemoptysis (i.e., pneumonia, mass, etc.) are identified, the diagnostic and therapeutic management for the detected lesion should follow (i.e., antibiotic treatment for a young patient with fever and a pulmonary opacity on CXR; further investigation in case of a middle-aged, smoker patient with a pulmonary opacity on CXR). If hemoptysis does not resolve or recurs, further treatment (medical, endovascular, and surgical) should be considered. On the other hand, it is advised to perform a MDCTA in case of a negative or nonlocalizing CXR, particularly when a lung cancer cannot be excluded (30).

If MDCTA reveals the cause of hemoptysis, the standard diagnostic and therapeutic management for the underlying condition should be performed. If MDCTA is negative and the episode of hemoptysis is resolving, it is possible to stop the investigation, whereas further examination such as bronchoscopy is warranted if the hemoptysis is persistent (33).

In case of a positive result at bronchoscopy, the flowchart follows the same indication as for the above-mentioned positive diagnostic tests. If a cause cannot be identified even at bronchoscopy, a cryptogenic hemoptysis should be considered. Clinicians should wait for a spontaneous resolution of bleeding 
in the first instance; however, in cases of recurrent bleeding an endovascular treatment could be advised (51).

\section{Conclusion}

Appropriate management of hemoptysis in different clinical settings is critical, especially in case of massive bleeding, which represents a life-threatening condition.

Imaging, especially MDCTA, has a major role in this context as it allows identification of a possible cause for the bleeding and guides eventual treatment, by providing a comprehensive and accurate assessment of the lung parenchyma, airways, and thoracic vessels.

Systemic bronchial and nonbronchial arteries are identifiable on MDCTA and play a major role in massive bleeding. A thorough knowledge of normal and variant anatomy of these arteries is crucial for the planning of endovascular embolization. Currently, arterial embolization is the most effective and minimally invasive procedure for treating massive and recurrent hemoptysis; pre-embolization MDCTA can reduce the bleeding recurrences and shorten the procedural time.

\section{Acknowledgements}

The authors thank Emiliano Visconti, MD, and Infante Amato, MD, for their contribution.

\section{Conflict of interest disclosure}

The authors declared no conflicts of interest.

\section{References}

1. Jeudy J, Khan AR, Mohammed TL, et al. ACR Appropriateness Criteria hemoptysis. J Thorac Imaging 2010; 25:W67-69.

2. Lordan JL, Gascoigne A, Corris PA. The pulmonary physician in critical care * Illustrative case 7: Assessment and management of massive haemoptysis. Thorax 2003; 58:814-819.

3. Ibrahim, WH. Massive haemoptysis: the definition should be revised. Eur Respir J 2008; 32:1131.

4. Yoon W, Kim JK, Kim YH, Chung TW, Kang HK. Bronchial and nonbronchial systemic artery embolization for life-threatening hemoptysis: a comprehensive review. Radiographics 2002; 22:1395-1409.

5. Jean-Baptiste E. Clinical assessment and management of massive hemoptysis. Crit Care Med 2000; 28:1642-1647.
6. Chun JY, Morgan R, Belli AM. Radiological management of hemoptysis: a comprehensive review of diagnostic imaging and bronchial arterial embolization. Cardiovasc Intervent Radiol 2010; 33:240250.

7. Andersen, PE. Imaging and interventional radiological treatment of hemoptysis. Acta Radiol 2006; 47:780-792.

8. Bruzzi JF, Remy-Jardin M, Delhaye D, Teisseire A, Khalil C, Remy J. Multi-detector row CT of hemoptysis. Radiographics 2006; 26:3-22.

9. Pump, KK. The bronchial arteries and their anastomoses in the human lung. Dis Chest 1963; 43:245-255.

10. Sirajuddin A, Mohammed TL. A 44-yearold man with hemoptysis: a review of pertinent imaging studies and radiographic interventions. Cleve Clin J Med 2008; 75:601-607.

11. McDonald, DM. Angiogenesis and remodeling of airway vasculature in chronic inflammation. Am J Respir Care Med 2001; 164:S39-S45.

12. Jaitovich A, Harmath C, Cuttica M. Pulmonary vein stenosis and hemoptysis. Am J Respir Crit Care Med 2012; 185:1023.

13. Youssef AI, Escalante-Glorsky S, Bonnet RB, Chen YK. Hemoptysis secondary to bronchial varices associated with alcoholic liver cirrhosis and portal hypertension. Am J Gastroenterol 1994; 89:1562-1563.

14. Park MS. Diffuse alveolar hemorrhage. Tuberc Respir Dis (Seoul) 2013; 74:151162.

15. Noë GD, Jaffé SM, Molan MP. CT and CT angiography in massive haemoptysis with emphasis on pre-embolization assessment. Clin Radiol 2011; 66:869-875.

16. Boulay F, Berthier F, Sisteron O, Gendreike $\mathrm{Y}$, Blaive B. Seasonal variation in cryptogenic and noncryptogenic hemoptysis hospitalizations in France. Chest 2000; 118:440-444.

17. Menchini L, Remy-Jardin M, Faivre JB, et al. Cryptogenic haemoptysis in smokers: angiography and results of embolization in 35 patients. Eur Respir J 2009; 34:1031-1039.

18. Hartmann IJ, Remy-Jardin M, Menchini L, et al. Ectopic origin of bronchial arteries: assessment with multidetector helical CT angiography. Eur Radiol 2007; 17:1943-1953.

19. Cauldwell EW, Siekert RG, Lininger R, et al. The bronchial arteries; an anatomic study of 150 human cadavers. Surg Gynecol Obstet 1948; 86:395-412.

20. Khalil A, Fartoukh M, Tassart M, Parrot A, Marsault C, Carette MF. Role of MDCT in identification of the bleeding site and the vessels causing hemoptysis. AJR Am J Roentgenol 2007; 188:W117-125.

21. Yoon YC, Lee KS, Jeong YJ, Shin SW Chung MJ, Kwon OJ. Hemoptysis: bronchial and nonbronchial systemic arteries at 16-detector row CT. Radiology 2005; 234:292-298.
22. Yildiz AE, Ariyurek OM, Akpinar E, Peynircioglu B, Cil BE. Multidetector CT of bronchial and non-bronchial systemic arteries. Diagn Interv Radiol 2011; 17:10 17.

23. Remy J, Lemaitre L, Lafitte JJ, Vilain MO, Saint Michel J, Steenhouwer F. Massive hemoptysis of pulmonary arterial origin: diagnosis and treatment. AJR Am J Roentgenol 1984; 143:963-969.

24. Khalil A, Parrot A, Nedelcu C, Fartoukh M, Marsault C, Carette MF. Severe hemoptysis of pulmonary arterial origin: signs and role of multidetector row CT angiography. Chest 2008; 133:212-219.

25. Hsiao EI, Kirsch CM, Kagawa FT, Wehner JH, Jensen WA, Baxter RB. Utility of fiberoptic bronchoscopy before bronchial artery embolization for massive hemoptysis. AJR Am J Roentgenol 2001; 177:861867.

26. Naidich DP, Funt S, Ettenger NA, Arranda C. Hemoptysis: CT-bronchoscopic correlations in 58 cases. Radiology 1990; 177:357-362.

27. McGuinness G, Beacher JR, Harkin TJ, Garay SM, Rom WN, Naidich DP. Hemoptysis: prospective high-resolution CT/ bronchoscopic correlation. Chest 1994; 105:1155-1162.

28. Hirshberg B, Biran I, Glazer M, Kramer MR. Hemoptysis: etiology, evaluation, and outcome in a tertiary referral hospital. Chest 1997; 112:440-444.

29. Revel MP, Fournier LS, Hennebicque AS, et al. Can CT replace bronchoscopy in the detection of the site and cause of bleeding in patients with large or massive hemoptysis? AJR Am J Roentgenol 2002; 179:1217-1224.

30. Herth F, Ernst A, Becker HD. Long-term outcome and lung cancer incidence in patients with hemoptysis of unknown origin. Chest 2001; 120:1592-1594.

31. Karmy-Jones R, Cuschieri J, Vallières E. Role of bronchoscopy in massive hemoptysis. Chest Surg Clin N Am 2001; 11:873-906.

32. Haponik EF, Fein A, Chin R. Managing life-threatening hemoptysis: has anything really changed? Chest 2000; 118:1431-1435.

33. Lee YJ, Lee SM, Park JS, et al. The clinical implications of bronchoscopy in hemoptysis patients with no explainable lesions in computed tomography. Respir Med 2012; 106:413-419.

34. Abal AT, Nair PC, Cherian J. Haemoptysis: aetiology, evaluation and outcome - a prospective study in a third-world country. Respir Med 2001; 95:548-552.

35. Khalil A, Soussan M, Mangiapan G, Fartoukh M, Parrot A, Carette MF. Utility of high-resolution chest CT scan in the emergency management of haemoptysis in the intensive care unit: severity, localization and aetiology. Br J Radiol 2007; 80:21-25. 
36. Remy-Jardin M, Bouaziz N, Dumont P, Brillet P-Y, Remy J. Bronchial and nonbronchial systemic arteries at multi-detector row CT angiography: comparison with conventional angiography. Radiology 2004; 233:741-749.

37. Morita $\mathrm{Y}$, Takase $\mathrm{K}$, Ichikawa $\mathrm{H}$, et al. Bronchial artery anatomy: preoperative 3D simulation with multidetector CT. Radiology 2010; 255:934-943.

38. Remy J, Remy-Jardin M, Bonnel F, Masson P. Spiral (helical) CT of tracheobronchial diseases. J Radiol 2000; 81:201-216.

39. Yoon W, Kim YH, Kim JK, Kim YC, Park JG, Kang HK. Massive hemoptysis: prediction of nonbronchial systemic arterial supply with chest CT. Radiology 2003; 227:232-238.

40. Sbano H, Mitchell AW, Ind PW, Jackson JE. Peripheral pulmonary artery pseudoaneurysms and massive hemoptysis. AJR Am J Roentgenol 2005; 184:1253-1259.

41. Hiller N, Lieberman S, Chajek-Shaul T, Bar-Ziv J, Shaham D. Thoracic manifestations of Behçet disease at CT. Radiographics 2004; 24:801-808.

42. Gossage JR, Kanj G. Pulmonary arteriovenous malformations. A state of the art review. Am J Respir Crit Care Med 1998; 158:643-661.
43. Remy J, Remy-Jardin M, Wattinne L, Deffontaines C. Pulmonary arteriovenous malformations: evaluation with CT of the chest before and after treatment. Radiology 1992; 182:809-816.

44. Ramakantan R, Bandekar VG, Gandhi MS, Aulakh BG, Deshmukh HL. Massive hemoptysis due to pulmonary tuberculosis: control with bronchial artery embolization. Radiology 1996; 200:691-694.

45. Mal H, Rullon I, Mellot F, et al. Immediate and long-term results of bronchial artery embolization for life-threatening hemoptysis. Chest 1999; 115:996-1001.

46. Tanaka N, Yamakado K, Murashima S, et al. Superselective bronchial artery embolization for hemoptysis with a coaxial microcatheter system. J Vasc Interv Radiol 1997; 8:65-70.

47. Wong ML, Szkup P, Hopley MJ. Percutaneous embolotherapy for life-threatening hemoptysis. Chest 2002; 121:95-102.

48. Shamji MF, Maziak DE, Shamji FM, Ginsberg RJ, Pon R. Circulation of the spinal cord: an important consideration for thoracic surgeons. Ann Thorac Surg 2003; 76:315-321.
49. Fernando HC, Stein M, Benfield JR, Link DP. Role of bronchial artery embolization in the management of hemoptysis. Arch Surg 1998; 133:862-866.

50. Swanson KL, Johnson CM, Prakash UB, McKusick MA, Andrews JC, Stanson AW. Bronchial artery embolization: experience with 54 patients. Chest 2002; 121:789-795.

51. Samara KD, Tsetis D, Antoniou KM, Protopapadakis C, Maltezakis G, Siafakas NM. Bronchial artery embolization for management of massive cryptogenic hemoptysis: a case series. J Med Case Rep 2011; 5:58.

52. Anuradha C, Shyamkumar NK, Vinu M, Surendra Babu NRS, Christopher DJ. Outcomes of bronchial artery embolization for life-threatening hemoptysis due to tuberculosis and post-tuberculosis sequelae. Diagn Interv Radiol 2012; 18:96-101.

53. Ianniello A, Carrafiello G, Nicotera P, Vaghi A, Cazzulani A. Endovascular treatment of a ruptured pulmonary artery aneurysm in a patient with Behcet's disease using the Amplatzer Vascular Plug 4. Korean J Radiol 2013; 14:283-286. 